\title{
RECENT PROGRESS IN OVARIAN CANCER THERAPY
}

\author{
Adeline Georgiana Staicu ${ }^{1^{\#}}$, Cristina Horescu ${ }^{1^{*}}$, Stefania Tudorache ${ }^{1,2^{\star}}$
}

\begin{abstract}
Ovarian cancer (OC) represents the leading cause of gynecological cancer deaths in women. It has a high variety of histological origins, molecular pathways and genetic mutations (BRCA1 and BRCA2 somatic/germinal mutations, Lynch genes, RADS1C, RADS1D etc.) that play a key role in treatment response and prognosis (for example, clear cell carcinoma is less responsive to standard therapy). An important percentage of patients (80\%) are diagnosed with metastatic disease, due to the lack of specific symptomatology and screening techniques leading to a dismal evolution. Standard treatment did not change drastically in decades consisting, nowadays, of cytoreductive surgery (debulking) and chemotherapy (a platinum-based agent, usually carboplatin and a taxane). Most patients experience complete response, but within a period of time, relapses occur and the cancerous cells may become platinum-resistant. This article depicts key clinical trials for many pharmacological agents including anti-angiogenesis drugs (bevacizumab, aflibercept, trebananib, pazopanib, sorafenib etc.), PARP-inhibitors (olaparib, niraparib), DNMT inhibitors (decitabine, azacytidine), various TKIs, DNA vaccines, oncolytic virus therapies and other agents.
\end{abstract}

Keywords: ovarian cancer, molecular agents, tyrosine kinase inhibitors.

1 University of Medicine and Pharmacy of Craiova, Faculty of Medicine, Romania

2 Emergency Clinical County Hospital of Craiova, Craiova, Romania

"Corresponding author: Stefania Tudorache (stefania.tudorache@gmail.com)

${ }^{\#}$ Authors have contributed equally to this work

Published online: 2 September 2020

\section{Introduction}

Ovarian cancer $(\mathrm{OC})$ is one of the most aggressive types of cancer in women, as early diagnosis is rarely performed, partly due to the lack of screening methods and to the apparently mild symptoms that can be easily confused with digestive problems (bloating, indigestion, constipation etc.).
Ovarian tumors present a high variety of histological origins and according to $\mathrm{WHO}$, they can be classified in: surface epithelial (serous, mucinous, endometrioid, clear cell, transitional, epithelial-stromal) $65 \%$, germ cell $(15 \%)$, sex cord-stromal (10\%), metastases such as Krukenberg tumors (5\%), miscellaneous; $90 \%$ of the malignant neoplasms being surface epithelial. The histological origin affects the treatment response and the evolution. Clear cell carcinoma (CCC), for example, is less responsive to standard therapy and as a consequence has a worse prognosis.

The current standard of care consists of cytoreductive surgery (debulking) and chemotherapy (a platinum-based agent, usually carboplatin and a taxane, usually 
paclitaxel intravenously). $80 \%$ of the patients have a complete response, but within a period of time most of them experience relapse after adjuvant chemotherapy. Furthermore, when the disease relapses, the cancerous cells become platinum-resistant. A recent study suggests that in high-grade serous cancer, stem cells (OCSC) - $\mathrm{ALDH}^{+}$persist after the first line therapy and contribute to the relapse. IL-6 appears to influence the platinum-induced OCSC. Second-line therapy options are topotecan, Iysosomal doxorubicin, gemcitabine etc. Bevacizumab (Avastin)-a monoclonal antibody that interferes with VEGF/VEGFR pathway and Olaparib - a PARP inhibitor are modern agents that are used along with chemotherapy or in palliative care. However, more than half of the patients die in less than 5 years(1). Many molecular pathways and genetic mutations have been studied and proven important in the course of the disease and play a key role in the potential treatment that can be implemented. Hereditary gene mutations account for $15-20 \%$ of cases (BRCA1 and BRCA2 somatic/germinal mutations - 75\%, BRIP1, Lynch genes, RADS1C, RADS1D etc.), the remainders are considered sporadic. Gene mutations result in overexpression of certain proteins, expression of abnormal or nonfunctional proteins that promote proliferation, selfrenewal, invasion and survival.

Epithelial OC is associated with the following molecular aberrations: TP53,
BRCA1 and 2, CDK12, RB1, NF1, PI3K/RAS/Notch/FOXM1 (in high-grade serous cancer); BRAF, NRAS, KRAS, ERBB2 (low-grade serous cancer); KRAS, HER2 (mucinous); ARID1A, PIK3CA, PTEN, CTNNB1, PPP2R1alfa (CCC); ARID1A, PIK3CA, PTEN, PPP2R1alfa, MMR deficiency (endometrioid). Nonepithelial OC is associated with FOXL2 (granulosa cell) and DICER1 (SertoliLeydig cell) aberrations.

\section{Therapies}

\section{Monoclonal antibodies}

Angiogenesis is an important factor in cancer growth and metastasis. Vascular endothelial growth factor VEGF $-A$ activates VEGFR-2, a tyrosine kinase receptor, and induces angiogenesis and vascular permeability. It is the target of bevacizumab.

\section{Anti VEGF antibodies}

Bevacizumab (Avastin) is a humanized recombinant monoclonal antibody that interferes with VEGFR ligand binding. It is used in combination with chemotherapy or as monotherapy $(15 \mathrm{mg} / \mathrm{kg}$ intravenously every 21 days). In recurrent epithelial $O C$ it has a response rate of 16-21\%. Bevacizumab significantly prolonged PFS in combination with carboplatin and paclitaxel, but with important gastrointestinal side effects (gastrointestinal perforation, bleeding). Up to date it is the most promising agent(2-4). 
Aflibercept represents a decoy receptor fusion protein, that targets VEGF-A, VEGF$B$ and placental growth factor (PIGF) and thus inhibits angiogenesis. Aflibercept reduces proliferation and tumor volume in other types of cancer. It has been studied in advanced chemo resistant epithelial OC yielding promising results (response rate $54 \%)$. Alongside docetaxel, it has proven a satisfying therapeutic benefit $(5,6)$.

\section{Anti CA125 antibodies}

Oregovomab is a specific monoclonal antibody against CA125 that forms circulating immune complexes capable of inducing an immune response. Oregovomab was studied as an adjuvant immunotherapy for first line chemotherapy (paclitaxel and carboplatin). There have been noted minimal toxicity and no prolonged PFS(7).

Abagovomab is a murine anti-idiotypic antibody that mimics CA125 protein and induces an immune response. Results in phase I and II trials proved abagovomab to be safe and tolerable, although, in a phase III study, the agent was not labeled clinically effective in regards to survival. Another study suggested that the immune system status is of high importance in obtaining a clinical response. Further studies are necessary for this agent(8).

Catumaxomab is a trifunctional monoclonal antibody that targets EpCAM positive cells by activating $T$ cells (CD3). It was studied as an ascites management agent in both platinum-resistant and - refractory EOC. Intraperitoneal treatment was safe and with tolerable toxicity(9).

Volociximab is an integrin inhibitor $m A b$ ( $\alpha 5 \beta 1)$ that provides anti-angiogenic effects and may hinder cell proliferation. A phase II trial proved the tolerability of the agent, but further studies are needed(10).

\section{Anti IGF-1R antibodies}

AMG 479 (Ganitumab) represents a mAb against IGF-1R that affects cell proliferation through PI3K/Akt and MAP kinase pathways inhibition. It has been studied in combination with carboplatin and paclitaxel in vitro, showing a synergic interaction and with cisplatin, in vivo, increasing its effect. There are more studies that need to be performed in order to assess the potential effectiveness of AMG 479(11).

\section{Other antibodies}

AMG 386 (trebananib) is a recombinant peptide-Fc fusion protein, which reduces angiogenesis by binding angiopietin- 1 and angiopoietin-2 and, thus, preventing the association to Tie2 receptor. AMG 386 has been studied in combination with paclitaxel, topotecan and carboplatin. The administration of trebananib resulted in manageable side effects such as hypokalemia. PFS was improved by 2.8 months, but OS remained virtually unchanged. The results show potential efficacity (7-9).

Farletuzumab is a humanized monoclonal antibody (immunoglobulin G, subtype 1 kappa) which possesses high receptor affinity and, supposedly, induces 
autophagy and inhibits Lyn kinase. Farletuzumab has been studied in phase IIII trials, alone or in association to paclitaxel and carboplatin, in patients with recurrent disease. The results showed an acceptable toxic profile with most of the symptoms linked to standard chemotherapy, but PFS was not significant $(10,11)$.

\section{Tyrosine kinase inhibitors}

\section{EGFR inhibitors}

Gefitinib, erlotinib and lapatinib are TKIs targeting EGFR and HER-2. Clinical trials investigated the response of a regimen composed of erlotinib in combination with conventional chemotherapeutic agents, rendering no improvements. A phase II trial assessed the effects of erlotinib and bevacizumab in patients with recurrent ovarian cancer. The results showed a high risk of severe gastrointestinal perforation(12).

A phase I trial evaluated lapatinib in patients with recurrent or persistent $O C$ in combination with carboplatin and yielded poor results and severe toxicity. Despite the overexpression of EGFR, HER-2 and EGFR targeted therapies have poor response(13).

\section{PDGFR and c-Kit inhibitors}

Imatinib is a TK selective inhibitor of $A B L$ kinase, BCR-ABL oncoprotein in CML, PDGFRs. It has been studied as monotherapy or in alongside docetaxel, paclitaxel, gemcitabine. The results showed no relevant response with no effect on PFS prolongation $(14,15)$.

\section{Multikinase Inhibitors of angiogenesis}

Pazopanib is an orally multitargetingTKI(VEGFR, PDGFR, FGFR, cKIT and RET) that exhibitsan additional anti-tumorigenic effect. In a randomized, double-blind phase III study (AGO OVAR 16), pazopanib (800 mg orally) was administered to the carboplatin and paclitaxel scheme. Results showed an improved PFS of 5.6 months, but no effect on OS. As adverse effects, hypertension, neutropenia, liver toxicity and diarrhea were reported. Another study in combination to topotecan rendered a 1.7fold drug exposure as opposed to monotherapy. This scheme would require future research(16).

Nintedanib is a triple angiokinase inhibitor (VEGFR, PDGFR, FGFR) which was part of a phase III clinical trial along with the administration of standard chemotherapy. Results showed a prolonged median PFS (from 16.6 to 17.3 months) and no effect on the OS. AEs prevailed in the hematologic and gastrointestinal sectors $(17,18)$.

Sorafenib is a multikinase inhibitor (VEGFR2, VEGFR3, PDGFR beta, Flt-3 and $\mathrm{C}$-kit), having an inhibitory action on RAS/RAF/MEK/ERK signaling pathway. In a phase II study in combination with conventional chemotherapy, patients achieved partial responses (3.4\%) and $34 \%$ experienced stable disease. Sorafenib in monotherapy confers modest activity and at the expense of notable toxicity. The most 
common AEs were hand-foot skin reaction and $\operatorname{rash}(19,20)$.

Dasatinib is a multitargeting $\mathrm{TKI}$, including Src (implication in platinumresistance), Lck, Bcr-Abl, c-Kit, PDGFR, FAK, EphA2. In recent studies, dasatinib along with paclitaxel, carboplatin, gefitinib, crizotinib had a synergic effect on regulating apoptosis, migration and invasion(21).

\section{Inhibitors of the main signaling pathways}

Rapamycin (mTOR) signaling pathway

$\mathrm{PI3K} / \mathrm{AKT} / \mathrm{mTOR}$ pathway mutations are present in clear cell ovarian cancer and endometroid adenocarcinoma accounting for proliferation and cell survival.

\section{Temsirolimus and everolimus}

Temsirolimus proved efficiency in endometrial cancer and has been included in clinical trials assessing its toxicity, safety and tolerability in OC. A phase II study showed modest results when it was used as monotherapy in persistent and relapsed disease. The noted adverse effects were fatigue, renal failure, pulmonary embolism, gastrointestinal symptoms. Temsirolimus was studied in a phase I clinical trial in combination with topotecan in advanced and recurrent $\mathrm{OC}$. The study has been terminated because of increased toxicity (myelosuppression). Another phase I study included temsirolimus along with carboplatin and paclitaxel and was proven effective and well tolerated. A phase $\mathrm{lb}$ clinical trial assessed tesmirolimus in combination with pegylated liposomal doxorubicin. The side effects were fatigue, mucositis and nausea. Temsirolimus was also studied in association to bevacizumab in advanced tumors and the most frequent adverse effects were thrombocytopenia and fatigue.

Everolimus was included in a phase I clinical trial consisting of a regimen composed of panitumumab and bevacizumab. The results showed a moderate activity. Adverse effects included hypertension, mucositis, electrolytic disorders(22).

\section{Inhibitors of DNA-repairing mechanisms} Polyadenosine diphosphate-ribose polymerase inhibitors

PARP enzymes catalyze the addition of ADP-ribose units to DNA, being of outmost importance in single-strand DNA breaks repairing. If the mechanism is defective, single-strand DNA breaks quickly lead to double-strand DNA breaks. BRCA mutations are sensitizers to DNA breaks. PARPi lead to the accumulation of DNA breaks and so resulting in antitumor activity.

Olaparib is a PARP-1, -2, -3 inhibitor that received FDA approval and is currently used as $4^{\text {th }}$ line treatment in patients with BRCA mutations.

Niraparib is a PARP-1, -2 inhibitor with affinity to PARP-2 that was also FDA approved for therapy in women with recurrent $\mathrm{OC}$ (regardless of BRCA type of mutation) who have previously been 
treated with platinum-based chemotherapy.

There were noted hematological (thrombocytopenia, anemia, neutropenia), respiratory and cardiovascular adverse effects(23).

\section{DNA methylation inhibitors}

DNA methylation (DNA methyltransferases) along with tumor suppressor genes inactivation can lead to oncogenesis. DNMT inhibitors (decitabine and azacytidine) have been evaluated in phase I/II trials as platinum-resistant $O C$ sensitizer, displaying a promising response. A study including decitabine, paclitaxel, carboplatin with cytokineinduced killer cells resulted in a prolonged PFS in platinum-resistant patients. Further studies are needed(24).

Histone deacetylase removes acetyl groups and can offer chemotherapeutic resistance, thus combing HDAC inhibitors with chemotherapy may provide anticancer effects.

Vorinostat has been analyzed in combination to paclitaxel, carboplatin and gemcitabine in recurrent platinum-sensitive OC, but the adverse effects imposed the termination of the study(25).

Ubiquitin-proteasome

pathway

mutations are involved in a variety of neoplasms.

Bortezomib efficacy has been under the assessment of clinical trials as monotherapy or in combination with carboplatin, paclitaxel, gemcitabine. A recent study enhanced the synergic effects of bortezomib and doxorubicin in OC by using nanocarriers in order to deliver the two agents(26).

\section{Immunotherapy}

\section{DNA Vaccine}

Follicle-stimulating hormone receptor (FSHR) is a transmembrane receptor expressed in healthy tissue (only in the granulosa cells of the selected follicle), $50 \%$ of OCs, endothelial cells of cancerous neoangiogenesis and metastasis. A new study designed a DNA vaccine to target FSHR and break immune tolerance starting from the principle that $\mathrm{OC}$ is an immunogenic disease. 2 types of vaccines were created using native or SynCon FSHR sequence. Both were capable of inducing a CD4 and CD8-dependent immune response, although CD8 $\mathrm{T}$ cells were the main players. There were observed important levels of IFNy, TNF- $\alpha$ and IL-2. SynCon vaccine yielded superior immune response in comparison to native vaccine. The final results showed an increase in survival by up to 3 times in $25 \%$ of mice. As AEs only infertility would be expected.(27).

\section{Oncolytic Virus Therapy}

OVs (oncolytic virus) are potent combatants in cancer therapy, showing encouraging results in other types of cancers such as melanoma. It has been observed that systemic administration of OVs results in an altered architecture of tumor vascularization implying furthermore a reduced perfusion with higher levels of 
hypoxia. This phenomenon would render negative disease evolution such as metastasis. A recent study tackled this aspect by administering an agent (3TSR) that stabilizes the vascularization prior to the treatment with NDV (oncolytic Newcastle disease virus). 3TSR is an agent that not only normalizes the vascularization, but also promotes tumor cells apoptosis and increases the tumor infiltrating capacities of leukocytes with no apparent toxicity. In combination with the OV, primary tumor size has considerably decreased as well as secondary lesions and the action of other agents that require good perfusion and oxygen levels would probably improve. This new strategy holds great promise for future studies(28).

\section{Conclusions}

Ovarian cancer is the first cause of gynecologic cancer deaths and presents in $80 \%$ of patients as an end stage disease. Despite the advances in surgery and chemotherapy, treatment efficacy remains almost unchanged in decades, hence the need for more studies and therapeutic agents.

\section{Conflict of interest}

The authors declare no conflict of interest.

\section{References}

1. Wang $Y$, Zong X, Mitra S, Mitra AK, Matei D, Nephew KP. IL-6 mediates platinum-induced enrichment of ovarian cancer stem cells. JCI insight. 2018;3(23). https://doi.org/10.1172/jci.insight. 122360

2. Itamochi H. Targeted therapies in epithelial ovarian cancer: Molecular mechanisms of action. World journal of biological chemistry. 2010;1(7):209-20. https://doi.org/10.4331/wjbc.v1.i7.209

3. Richardson DL, Backes FJ, Seamon LG, Zanagnolo V, O'Malley DM, Cohn DE, et al. Combination gemcitabine, platinum, and bevacizumab for the treatment of recurrent ovarian cancer. Gynecologic oncology.2008;111(3):461-6.

https://doi.org/10.1016/j.ygyno.2008.08.011

4. Perren TJ, Swart AM, Pfisterer J, Ledermann JA, Pujade-Lauraine $E$, Kristensen $G$, et al. A phase 3 trial of bevacizumab in ovarian cancer. The New England journal of medicine. 2011;365(26):2484-96. https://doi.org/10.1056/NEJMoa1103799

5. Colombo N, Mangili G, Mammoliti S, Kalling M, Tholander B, Sternas L, et al. A phase II study of aflibercept in patients with advanced epithelial ovarian cancer and symptomatic malignant ascites. Gynecologic oncology. 2012;125(1):42-7. https://doi.org/10.1016/i.ygyno.2011.11.021

6. Coleman RL, Duska LR, Ramirez PT, Heymach JV, Kamat AA, Modesitt SC, et al. Phase 1-2 study of docetaxel plus aflibercept in patients with recurrent ovarian, primary peritoneal, or fallopian tube cancer. The Lancet Oncology. 2011;12(12):1109-17. https://doi.org/10.1016/S1470-2045(11)70244-3

7. Berek JS, Taylor PT, Gordon A, Cunningham MJ, Finkler N, Orr J, Jr., et al. Randomized, placebocontrolled study of oregovomab for consolidation of clinical remission in patients with advanced ovarian cancer. Journal of clinical oncology : official journal of the American Society of Clinical Oncology. 2004;22(17):3507-16.

https://doi.org/10.1200/JCO.2004.09.016

8. Battaglia A, Fossati M, Buzzonetti A, Scambia G, Fattorossi A. A robust immune system conditions the response to abagovomab (anti-idiotypic monoclonal antibody mimicking the CA125 protein) vaccination in ovarian cancer patients. Immunology letters. 2017;191:35-9.

https://doi.org/10.1016/i.imlet.2017.09.006

9. Kurbacher CM, Horn O, Kurbacher JA, Herz S, Kurbacher AT, Hildenbrand R, et al. Outpatient Intraperitoneal Catumaxomab Therapy for Malignant Ascites Related to Advanced Gynecologic Neoplasms. The oncologist. 2015;20(11):1333-41. https://doi.org/10.1634/theoncologist.2015-0076

10. Bell-McGuinn KM, Matthews CM, Ho SN, Barve M, Gilbert L, Penson RT, et al. A phase II, single-arm study of the anti- $\alpha 5 \beta 1$ integrin antibody volociximab as 
monotherapy in patients with platinum-resistant advanced epithelial ovarian or primary peritoneal cancer. Gynecologic oncology. 2011;121(2):273-9. https://doi.org/10.1016/j.ygyno.2010.12.362

11. Beltran PJ, Calzone FJ, Mitchell P, Chung YA, Cajulis E, Moody G, et al. Ganitumab (AMG 479) inhibits IGFII-dependent ovarian cancer growth and potentiates platinum-based chemotherapy. Clinical cancer research : an official journal of the American Association for Cancer Research. 2014;20(11):294758.

https://doi.org/10.1158/1078-0432.CCR-13-3448

12. Nimeiri HS, Oza AM, Morgan RJ, Friberg G, Kasza K, Faoro L, et al. Efficacy and safety of bevacizumab plus erlotinib for patients with recurrent ovarian, primary peritoneal, and fallopian tube cancer: a trial of the Chicago, $\mathrm{PMH}$, and California Phase II Consortia. Gynecologic oncology. 2008;110(1):49-55. https://doi.org/10.1016/i.ygyno.2008.02.009

13. Kimball KJ, Numnum TM, Kirby TO, Zamboni WC, Estes JM, Barnes MN, et al. A phase I study of lapatinib in combination with carboplatin in women with platinum sensitive recurrent ovarian carcinoma. Gynecologic oncology. 2008;111(1):95-101. https://doi.org/10.1016/j.ygyno.2008.07.001

14. Patel BB, He YA, Li XM, Frolov A, Vanderveer L, Slater $C$, et al. Molecular mechanisms of action of imatinib mesylate in human ovarian cancer: a proteomic analysis. Cancer genomics \& proteomics. 2008;5(3-4):137-49.

15. Juretzka M, Hensley ML, Tew W, Konner J, Aghajanian $C$, Leitao $M$, et al. A phase 2 trial of oral imatinib in patients with epithelial ovarian, fallopian tube, or peritoneal carcinoma in second or greater remission. European journal of gynaecological oncology. 2008;29(6):568-72.

16. Richardson DL, Sill MW, Coleman RL, Sood AK, Pearl ML, Kehoe SM, et al. Paclitaxel with and without pazopanib for persistent or recurrent ovarian cancer: a randomized clinical trial. JAMA oncology. 2018;4(2):196-202.

https://doi.org/10.1001/jamaoncol.2017.4218

17. Khalique S, Banerjee S. Nintedanib in ovarian cancer. Expert opinion on investigational drugs. 2017;26(9):1073-81.

https://doi.org/10.1080/13543784.2017.1353599

18. Awasthi N, Schwarz RE. Profile of nintedanib in the treatment of solid tumors: the evidence to date. OncoTargets and therapy. 2015;8:3691-701. https://doi.org/10.2147/OTT.S78805

19. Matei D, Sill MW, Lankes HA, DeGeest K, Bristow RE, Mutch $D$, et al. Activity of sorafenib in recurrent ovarian cancer and primary peritoneal carcinomatosis: a gynecologic oncology group trial. Journal of clinical oncology : official journal of the American Society of Clinical Oncology. 2011;29(1):69-75. https://doi.org/10.1200/JCO.2009.26.7856
20. Welch SA, Hirte HW, Elit L, Schilder RJ, Wang L, Macalpine $\mathrm{K}$, et al. Sorafenib in combination with gemcitabine in recurrent epithelial ovarian cancer: a study of the Princess Margaret Hospital Phase II Consortium. International journal of gynecological cancer : official journal of the International Gynecological Cancer Society. 2010;20(5):787-93. https://doi.org/10.1111/IGC.0b013e3181e273a8

21. Thibault B, Jean-Claude B. Dasatinib + Gefitinib, a non platinum-based combination with enhanced growth inhibitory, anti-migratory and anti-invasive potency against human ovarian cancer cells. Journal of ovarian research.2017;10(1):31.

https://doi.org/10.1186/s13048-017-0319-2

22. Gasparri ML, Bardhi E, Ruscito I, Papadia A, Farooqi AA, Marchetti C, et al. PI3K/AKT/mTOR Pathway in Ovarian Cancer Treatment: Are We on the Right Track? Geburtshilfe und Frauenheilkunde. 2017;77(10):1095-103.

https://doi.org/10.1055/s-0043-118907

23. Mullen MM, Kuroki LM, Thaker PH. Novel treatment options in platinum-sensitive recurrent ovarian cancer: A review. Gynecologic oncology. 2019;152(2):416-25. https://doi.org/10.1016/i.ygyno.2018.10.023

24. Zhang Y, Mei Q, Liu Y, Li X, Brock MV, Chen M, et al. The safety, efficacy, and treatment outcomes of a combination of low-dose decitabine treatment in patients with recurrent ovarian cancer. Oncoimmunology.2017;6(9):e1323619. https://doi.org/10.1080/2162402X.2017.1323619

25. Matulonis U, Berlin S, Lee H, Whalen C, Obermayer E, Penson R, et al. Phase I study of combination of vorinostat, carboplatin, and gemcitabine in women with recurrent, platinum-sensitive epithelial ovarian, fallopian tube, or peritoneal cancer. Cancer chemotherapy and pharmacology. 2015;76(2):417-23. https://doi.org/10.1007/s00280-015-2813-9

26. Wang L, Shi C, Wright FA, Guo D, Wang X, Wang D, et al. Multifunctional Telodendrimer Nanocarriers Restore Synergy of Bortezomib and Doxorubicin in Ovarian Cancer Treatment. Cancer research. 2017;77(12):3293-305.

https://doi.org/10.1158/0008-5472.CAN-16-3119

27. Perales-Puchalt A, Wojtak K, Duperret EK, Yang X, Slager AM, Yan J, et al. Engineered DNA Vaccination against Follicle-Stimulating Hormone Receptor Delays Ovarian Cancer Progression in Animal Models. Molecular therapy : the journal of the American Society of Gene Therapy. 2019;27(2):314-25. https://doi.org/10.1016/j.ymthe.2018.11.014

28. Matuszewska K, Santry LA, van Vloten JP, Au Yeung AW, Major PP, Lawler J, et al. Combining Vascular Normalization with an Oncolytic Virus Enhances Immunotherapy in a Preclinical Model of AdvancedStage Ovarian Cancer. Clin Cancer Res. 2019;25(5):1624-1638. https://doi.org/10.1158/1078-0432.CCR-18-0220 